\title{
Uptake of genetic testing for cancer predisposition
}

\author{
D Gareth R Evans, Eamonn R Maher, Rhona Macleod, D Rhodri Davies, David Craufurd
}

\begin{abstract}
Although there has been much debate about the uptake and effects of predictive testing for common cancers, such as breast and colon cancer, little has been published on the more classical tumour predisposing conditions, such as von Hippel-Lindau disease and familial adenomatous polyposis. Since 1990 the genetics departments in Manchester and Cambridge have had a genetic register for cancer predisposing syndromes and presymptomatic testing for these conditions has been offered once this has become possible. To investigate the factors that might influence uptake of genetic testing in familial cancer syndromes we have reviewed our experience. Demand for predictive testing has generally been high, but men had a lower uptake $(\mathbf{7 7 \%})$ than a comparable group of women (93\%) (p<0.01).

( $\mathcal{F}$ Med Genet 1997;34:746-748)
\end{abstract}

Keywords: DNA predictive testing; cancer; FAP; vHL; NF2

Recent advances in molecular genetics have opened up the possibility of definitive presymptomatic testing for many people at risk of inherited cancer predisposing syndromes, yet little has been written to date about the ethical issues and counselling problems associated with offering such tests to this particular group of patients. In some conditions, such as neurofibromatosis type 1 (NF1) where phenotypic abnormalities sufficient to allow a clinical diagnosis are nearly always present by 5 years of age,${ }^{1}$ the need for predictive genetic testing is minimal. However, in others, such as familial adenomatous polyposis (FAP), neurofibromatosis type 2 (NF2), and von Hippel-Lindau disease (vHL), the phenotype is variable and diagnostic features may not be present until well into adult life. ${ }^{2-4}$ Predictive testing for the genes which predispose to these disorders may therefore have an important role in the identification of people most likely to benefit from screening aimed at the early detection and prevention of malignant lesions.

Screening programmes of this type are usually offered to people at $50 \%$ prior risk of these disorders, and the test procedures involved range from mild (for example, indirect ophthalmoscopy with mydriasis, MRI scanning of the brain) to considerable invasiveness (sigmoidoscopy/colonoscopy). However, the performance of these tests has not been subjected to the same degree of ethical scrutiny as DNA predictive tests. Some of these tests offer clear benefits for the at risk people concerned, in that they are intended to identify premalignant lesions, such as adenomatous polyps (FAP), vestibular schwannomas (NF2), or retinal angiomas (vHL). These can be treated and further disability prevented. Other abnormalities detected by some of these screening procedures, such as congenital hypertrophy of the retinal pigment epithelium (CHRPE) in FAP, posterior lenticular opacities in NF2, and multiple pancreatic cysts in vHL, merely indicate that the person concerned has inherited the mutated gene, without there being any immediate clinical benefit to the person. Unlike favourable DNA predictive tests, failure to identify these clinical abnormalities does not provide the same level of reassurance.

In breast cancer families (BRCA1/2, p53), where no such phenotypic markers exist, there has been much more emphasis on the use of DNA predictive tests, but we report here for the first time our experience of the uptake of predictive testing for FAP, vHL, and NF2, as well as some preliminary data from p53 mutation positive families with Li-Fraumeni syndrome.

\section{Patients and methods}

Uptake of genetic testing was assessed in two major regional genetics referral centres with a particular interest in cancer predisposition. Both centres have had a systematic approach to ascertaining affected and at risk subjects with FAP and vHL and one centre has also applied this approach to NF2. All at risk subjects from families in which genetic testing has been possible were approached through their general practitioners and by direct letter. Failure to respond to these approaches was taken as a decision to decline testing (at least for the time being) and this assumption was usually backed up by verbal confirmation from other family members. All those who responded to the invitation had at least two genetic counselling sessions, although frequently the first of these consisted of a discussion about genetic testing at a home visit by a genetic associate. Uptake of testing was assessed in three groups: children considered too young to participate in screening programmes (FAP $<10$ years, NF2 $<10$ years, vHL $<5$ years); other children of screening age (that is, 10-16 years for FAP); and adults. Differences in uptake were assessed between conditions and as a factor of age and sex.

\section{Results}

There were a total of 101 affected families with FAP on the two registers. Predictive testing was possible in 74 of these. The uptake of 
predictive testing for FAP in adults is shown in table 1. Many of the males had previously refused to have endoscopic screening and were grateful for a test which could determine whether they really required this. Two of the seven females who were not tested could not be contacted as their affected male relatives who were of Asian origin refused to allow an approach. Contact in a further white family has only been made possible since the death of the affected patriarchal figure.

Seventy-five presymptomatic tests for FAP were carried out on children aged 10-16 years in the 5.5 years up to December 1995. Where contact has been established with parents there have only been three children (from one affected father) who have not proceeded to testing. Wherever possible the child has been actively involved in the decision. A further five children eligible for testing were unavailable as their parents refused contact with the departments. However, there may be a number of other families in the two regions who have purposefully avoided contact with the departments, and in these families the uptake of testing may be lower. There are also currently 63 children aged under 10 years who are at $50 \%$ risk of FAP. Although we have had some pressure to perform tests on children under 10 years of age, parents have usually been willing to wait once they have received adequate information and counselling.

There were 23 NF2 families on the North Western Regional Genetic Register containing 37 affected subjects. Testing on regional families has been extended to members living outside the region. Total uptake of predictive testing for NF2 and vHL is shown in table 1 ; of the adults with vHL, $3 / 6$ subjects aged $16-20$ years declined testing, whereas $37 / 39$ aged over 20 years proceeded. In the $14 \mathrm{NF} 2$ families in which testing was possible, $14 / 14$ children aged 10-16 years have been tested after parental counselling which usually involved the child. One child aged 8 years was tested at the same time as her sibs.

Nineteen families with Li-Fraumeni or Li-Fraumeni-like syndrome were identified as having p53 germline mutations. ${ }^{5}$ In five of these families genetic counselling including discussion of presymptomatic testing has been offered to those at risk. Twelve of the 16 adults at $50 \%$ risk have been seen for genetic counselling in Manchester and the remaining people

Table 1 Uptake of DNA predictive testing in cancer prone syndromes in two genetic centres

\begin{tabular}{llllll}
\hline & & \multicolumn{2}{l}{ Age $(y)$} & & \\
\cline { 3 - 4 } Disease & Prescreen & $5-9$ & $10-16$ & Adult males & Adult females \\
\hline FAP & $0 / 63$ & & $75 / 78$ & $50 / 66$ & $69 / 74^{\star}$ \\
vHL & $0 / 6$ & $6 / 18$ & $2 / 4$ & $19 / 23$ & $20 / 22$ \\
NF2 & $1 / 12$ & & $14 / 14$ & $10 / 14$ & $23 / 25$ \\
LFS & $0 / 3$ & & $4 / 8$ & $2 / 8$ & $2 / 8$ \\
Total & $1 / 81$ & $6 / 18$ & $91 / 96(95 \%)$ & $79 / 103(77 \%)$ & $112 / 121(93 \%)$ \\
\end{tabular}

^Two women who were not contactable through their father were excluded. LFS $=$ Li-Fraumeni syndrome; these results are excluded from the totals as the issues regarding uptake and screening are different.

Male/female differences in uptake:

Total: $\chi^{2}=9.92, p<0.01$.

FAP: $\chi^{2}=7.05, \mathrm{p}<0.01$ are aware of the possibility of genetic tests. The parents of eight of the 11 children at $50 \%$ risk have also received genetic counselling. Testing has been offered to the adults, but only $4 / 16$ have decided to proceed after going through a predictive test counselling protocol based on that designed for Huntington's disease. ${ }^{6} \mathrm{Al}-$ though no specific offer of a genetic test was made to the parents of children at $50 \%$ risk, we have come under extreme pressure to undertake testing in four children aged 10-12 years. After at least three counselling sessions (involving in one case a child psychologist) and with involvement of the child in decision making, tests have been performed on two children from different families, neither of whom had living sibs. Another two sibs at $50 \%$ risk were tested anonymously as child A and child B. Both parents were terminally ill with cancer and were only to be told that either both children carried the mutation or both did not; if one child carried the mutation and the other did not, the affected child would not be revealed. Three of these four children received low risk results. Twelve months after testing there have been no adverse consequences and the situation has not worsened in the case of the high risk result. Although testing was carried out in Manchester, three out of four of the presymptomatic tests were requested from two other genetic centres.

\section{Discussion}

There have been no systematic studies to assess uptake of predictive testing for most of these cancer predisposing genes. This report represents a first attempt to document the differences in uptake both within and between these various syndromes. While the principle of nondirective genetic counselling is widely accepted and advocated, it is possible that the high uptake of testing in our FAP and NF2 families may reflect a more directive approach because of the importance of subsequent screening for those identified as gene carriers. Nonetheless, the negative implications of an unfavourable test result in terms of life insurance and the psychological impact of knowing about carrying a gene which may not cause problems for a number of years were made clear to all patients contemplating a test. ${ }^{78}$ Uptake was particularly high in at risk subjects who were already taking part in ongoing screening programmes, for whom the benefit of discontinuing screening in the event of a favourable result was seen as a major bonus.

Previous studies have also found a high uptake of predictive testing for FAP. Only $3 / 53$ people aged over 10 years were not tested in an early study from the West Midlands'; two of these were aged between 10 and 16 years, where the parents refused testing. There are no previous population based studies of uptake for NF2.

Similarly, high levels of uptake of genetic testing had been predicted for breast cancer predisposition from theoretical interviews and from testing in a single family. ${ }^{10}{ }^{11}$ However, early results from our own studies and another UK study of 32 at risk subjects suggest a lower 
uptake, particularly among men, once people have had time to reflect on the real consequences of a high risk result. ${ }^{12}{ }^{13}$ Early indications would suggest that for $\mathrm{Li}$-Fraumeni syndrome, uptake may not be much different from that for Huntington's disease. ${ }^{6}$ Only $12 / 48$ people at risk of carrying a p53 mutation have entered a testing programme in a US study ${ }^{14}$ and our results for adults are very similar. It is important to distinguish between talking about a potential genetic test and the actual uptake of a real one. Our recent report of a large family with Li-Fraumeni syndrome highlighted this point; four subjects at $50 \%$ risk gave blood for research purposes stipulating that they would want to know the result if the gene test became available, but once they were offered a definitive test all four withdrew from testing. ${ }^{15}$

In this study we have found a generally lower uptake of testing by males. While such a finding may not be surprising for BRCA1 testing, because males are not at particularly high risk of cancer as a result of carrying a BRCA1 mutation, they are at similar risks to females for FAP, NF2, and vHL. Similarly, uptake of predictive testing for Huntington's disease is lower in men than in women. ${ }^{6}$ It is possible that males are less interested in health matters, since they are also much less likely to adhere to screening programmes. In conditions where screening is vital, such as FAP, the predictive DNA test may be the only means of persuading a man to undergo endoscopy. It is also interesting to note that of the families where it has not been possible to contact some of the at risk relatives, this has usually been because of the antipathy of an affected male patriarchal figure. We have also experienced similar antipathy in the unaffected widowers of affected females, particularly in relation to the risks for their own children. In some cultures with a tradition of arranged marriages, there may also be a temptation to conceal the genetic implications for female relatives, who may otherwise become less eligible as potential spouses.

The debate about testing in childhood is straightforward when the risk of becoming clinically affected during childhood is close to zero. ${ }^{16}$ As this is the case with BRCA1 and HNPCC, there are few potential benefits of testing a child, and testing should be resisted. Insurance companies do not necessarily weight policies against people from hereditary breast/ ovarian cancer or HNPCC families as the forms do not usually tease out sufficient information. However, once a BRCA1 or HNPCC mutation has been found there would be little option but to declare this and suffer the financial penalties. Thus, a child whose autonomy had been removed could be disadvantaged later and may even have a reasonable case to take legal action against their parent or doctor. However, for FAP (as for Huntington's dis- ease) insurance may anyway be difficult to obtain without a genetic test to show that the person has not inherited the family mutation. As FAP, NF2, and vHL have clinical implications in childhood, testing may often be justified because the benefits of withdrawal from sometimes unpleasant screening tests are clear. It is clearly preferable for the child also to be involved to some extent in the decision if at all possible. The situation for Li-Fraumeni syndrome is more complex as no accepted screening protocol exists and tangible benefit to the child is less easy to show. We would not advocate testing in childhood, but there may be circumstances where testing could be undertaken on a case by case basis after full evaluation of the situation for each child at risk.

The higher levels of uptake in eligible children (FAP, NF2, vHL) aged 10-16 years (95\%) compared with adults (85\%) almost certainly reflect parental demand and the inability of children to understand fully the issues involved. However, it is difficult to see how counselling could be improved to achieve uptake rates which more accurately reflect the decisions likely to be made by these people as adults. Thus, if testing is to be offered at all in this age group, some degree of future dissatisfaction should be anticipated.

We are grateful for the help of Dr P Lunt and Dr R Eeles who gave us information relating to their counselling of family members.

1 Huson SM, Harper PS, Compston DAS. Von Recklinghausen neurofibromatosis. Brain 1988;111:1355-81.

2 Evans DGR, Huson S, Donnai D, et al. A clinical study of type 2 neurofibromatosis. $Q \mathcal{F} M$ Med 1992;84:603-18.

3 Evans DGR, Guy SP, Armstrong J, et al. Non penetrance and late appearance of polyps in families with familial adenomatous polyposis. Gut 1993;34:1389-93.

4 Maher ER, Iselius L, Yates JRW, et al. Von Hippel Lindau disease: a genetic study. $\mathcal{F}$ Med Genet 1991;28:443-7.

5 Varley JM, McGown G, Thorncroft M, et al. Germline mutations of TP53 in Li Fraumeni families: an extended study of 39 families. Cancer Res (in press).

6 Craufurd D, Kerzin-Storrer L, Dodge A, Harris R. Uptake of presymptomatic predictive testing for Huntington's disease. Lancet 1989;ii:603-5.

7 Eeles R, El-Abdi S, Sodha H, Varley J, Evans DGR, Pritchard-Jones K. Predictive p53 testing in children: a suggested model. In preparation.

8 suggested model. In preparation. 224-7.

9 MacDonald F, Morton DG, Rindl PM, et al. Predictive diagnosis of familial adenomatous polyposis with linked DNA markers: population based study. BMF 1992;304: 869-72.

10 Struewing JP, Lerman C, Kase RG, Giambaressi TR, Tucker MA. Anticipated uptake and impact of genetic testing in hereditary breast and ovarian cancer families. Cancer Epidemiol Biomarkers Prev 1995;4:169-73.

11 Lynch HT, Watson P, Conway TA, et al. DNA screening for breast/ovarian cancer susceptibility based on linked markers. Arch Intern Med 1993;153:1979-87.

12 Binchy A, Evans DGR, Eng C, Hendy-Ibbs P, Ponder BAJ, Craufurd D. Factors influencing uptake of predictive testing for BRCA1 in five large BRCA1 linked families. (Submitted.)

13 Watson M, Murday V, Lloyd S, Ponder B, Averill D, Eeles $R$. Genetic testing in breast/ovarian cancer (BRCA1) famiR. Genetic testing in breasto

14 Schneider KA, Patenaude AF, Garber JE. Testing for cancer Schneider KA, Patenaude AF, Garber JE. Testing for

15 Varley JM, Tricker KJ, Thorncroft M, et al. An extended Li Varley JM, Tricker KJ, Thorncroft $\mathrm{M}$, et al. An extended $\mathrm{Li}$ Fraumeni kindred with gastric carcinoma and a

16 The genetic testing of children. Report of a Working Party of the Clinical Genetics Society (UK). Chairman Dr A Clarke. F Med Genet 1994;31:785-97. 